\title{
A Computational View of the Historical Controversy on Animal Electricity
}

\author{
Massimiliano Zaniboni \\ Department of Biosciences, and Center of Excellence for Toxicological Research (CERT), \\ University of Parma, Parma, Italy \\ Email: massimiliano.zaniboni@unipr.it
}

Received August 27 $7^{\text {th }}, 2012$; revised September $24^{\text {th }}, 2012$; accepted October $10^{\text {th }}, 2012$

\begin{abstract}
A scientific controversy retains often some controversial sides after its fundamentals have well been explained. This is particularly true for the controversy that arose in Italy in the second half of the eighteen century between the anatomist Luigi Galvani, and the physicist Alessandro Volta, around the intrinsic nature of nerve and muscular function. The two scientists were providing, almost simultaneously from the University of Bologna and Pavia respectively, two quite different explanations for the property of muscles of being electrically excitable and contract as a consequence. Science seemed then to touch the very intrinsic mechanism of living processes. Despite the fact that one of the two explanations was explaining better than the other, the weaker mechanism won the battle at the time. The biophysical mechanism of nerve excitability has then been clarified in 1950 by Hodgkin and Huxley, who later won the Nobel Prize for their work. They unequivocally showed that Galvani was right and Volta quite wrong. Only specialists though notice that the Galvani-Volta controversy is frequently still thought wrong in schools. In this brief essay I want to show how easy-to-handle computer models can unveil where the subtle source of the controversy was hidden, and how an interdisciplinary approach can help drawing light into the multiple aspects of this extraordinary story.
\end{abstract}

Keywords: Animal Electricity; Galvani-Volta Controversy; Computational Physiology; Excitability

\section{Introduction: The Controversy}

The dispute between Galvani and Volta (Figure 1) for the explanation of muscular excitability has been discussed in very detailed articles and essays (Bernardi, 2001; Bresadola, 2008; Piccolino, 1997; Piccolino, 1998) and I will give here only a brief and necessarily partial report of it. In the middle of eighteen century the "art of electricity" was moving its first steps as a real science; interestingly, the beginning of this science coincides with the beginning of the science of bio-electricity. William Harvey (1578-1657) had already shown in its "De Motu Cordis" (On the Motion of the Heart) the basic mechanism of the blood circulation through vessels, and a circulation of the same type was believed to move some "animal spirits" through the nerves. The eclectic Swiss anatomist Albrecht Von Haller (1708-1777) was one of the first scientists disputing this view and proposing, instead, the concept of an "irritability", intrinsic to the very nature of nerves and muscles. These were "irritated", in the sense that they were in a state of some high potential energy (still unknown at that time), that some also-unknown mechanism was able to free after stimulation. Leopoldo Caldani (1725-1813), an eminent Italian physiologist from the University of Padova, was one of the most influential followers of the Haller's theory. I note in passing that Haller's and Caldani's studies were somewhat well aligned with the eighteen century's zeitgeist and, not surprisingly, strongly opposed by the many, e.g. the Scottish physician Robert Whytt (1714-1766), who thought they were denying the role of soul in nervous activity, and favouring a mere materialistic view of these processes. At those times there was a constant and fruitful exchange between disciplines; "Experimental Philosophy" was the way the English chemist, theologian and historian Joseph Priestley (17331804) was calling his pioneeristic studies on electricity; and it was only after reading Haller's works that the political theorist, politician, scientist, musician, satirist, civic activist, statesman, diplomat Benjamin Franklin (1706-1790) started his seminal studies on electricity. Electrostatic machines started to appear on the working table of anatomists, chemists, and physicists, and allowed them to produce large amount of electrical charge. This could be stored in Leyden jars ("miraculous", in the words of Priestley), smart and beautiful electrical capacitors which made this charge usefully available for their experiments with wires, metal plates and animal preparations. What these early experiments showed was that there definitely was a link between electricity and the ability of nerves and muscles to respond to stimuli, conduct them, and contract.

In the second half of the century, Luigi Galvani was conducting his experiments on the so called "frog preparation", where the spinal cords, crural nerves, and lower limbs of the little amphibian, were dissected as a unit. One of the Galvani's findings, achieved by a fortunate chance, was that, when the two extremes of a bi-metallic arc were made to touch the two extremities of the preparation, the muscle contracted (Figure 2). Galvani knew well Haller's and Caldani's works: he concluded that nerves were electrically charged, and that the bi-metallic arc only let this charge flow, excite the nerve, and contract the muscle; detailed mechanisms were still to uncover but the principle seemed to be found. The fact that a bi-metallic arc was required and that a simple one-metallic arc did not the job was initially not explained by Galvani. He performed several ver- 

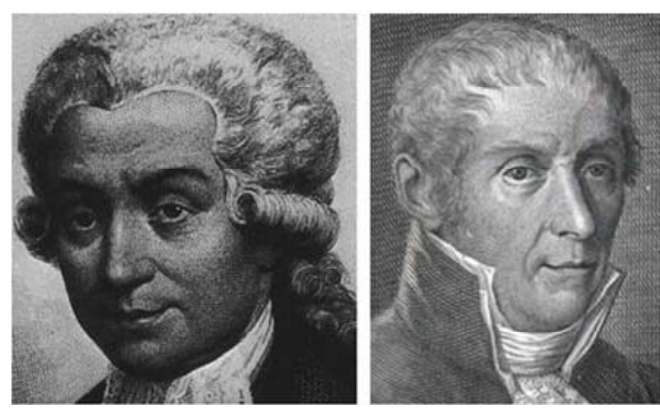

Figure 1.

Luigi Galvani, anatomist from the University of Bologna (1737-1798), and Alessandro Volta, physicist from the University of Pavia (1745- 1827).

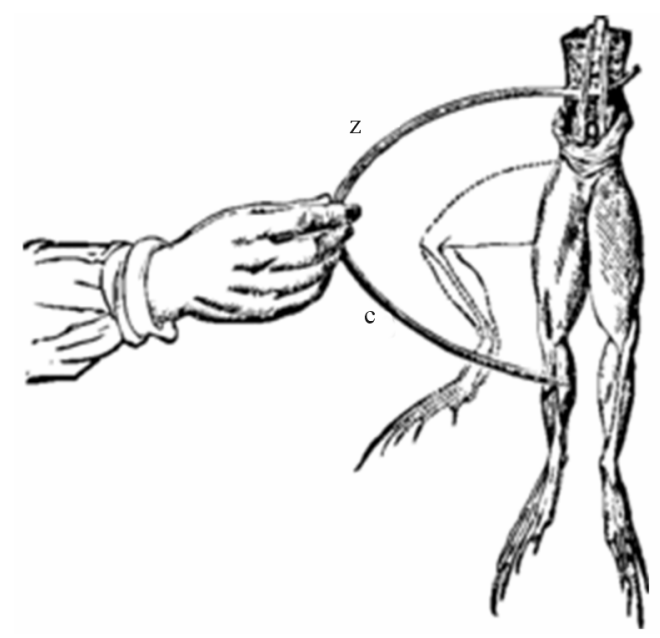

Figure 2.

The bimetallic-arc experiment. When an arc, made half of copper and half of zinc, touches the extremities of the frogpreparation, the limb suddenly contracts.

sions of this experiment as well as many others, that he lately detailed in his "De viribus electricitatis in motu muscularis", published in 1791; for the purpose of this paper, I will focus on this one only. The bi-metallic nature of the arc was indeed the very point were Galvani's theory was disputed by Alessandro Volta. His discovery that piles, made of plates of two different metals separated by salted solutions (Voltaic pile), were able to generate differences in electrical potential (measured after his name in Volts) and thus able to drive electrical current flow, seemed perfect to take the Galvani's explanation and turn it upside down. There was no animal electricity as Galvani claimed, no irritability nor any potential energy stored in tissues; only the bi-metallic arc in Galvani's experiments was a rudimentary Voltaic pile, making electrical current flow through muscles and providing the force needed for contraction. The mechanism responsible for contraction was obscure to both scientists and was not object of the dispute anyway. A complete account of the controversy can be found in a recent book of Piccolino and Bresadola (Piccolino \& Bresadola, 2003). I want here to point, as these authors detail in their work, to the persisting misinterpretation of the controversy: both in schools and in popular scientific handbooks, it is still thought frequently that the wrong Galvani's hypothesis of an "animal electricity" had only served the successful Volta's theory of an external force driving nerve conduction and muscle contraction, which eventually started the immense technological revolution of electricity. In this article I will shortly describe the theory of nerve excitability as it has been explained in the 20th century. The explanation can be summarized into an equation system that can easily be solved by means of tools from modern computer science. A compact computational view of one of the Galvani-Volta's experiments can help teachers and students to better dissect the critical points of this instructive controversy.

\section{The Hodkin and Huxley Theory of Excitability}

As I have summarized in the introduction, whereas Galvani retained that the source of "animal electricity" was inside the animal, Volta's explanation put this source externally. The impact that Volta's discoveries had on science and not only on science (think what electricity has done to human history), is one of the reasons, not the only one, for its success over the dispute with Galvani. Excitability has been explained in detail through a bio-physical model developed by Hodgkin and Huxley (Figure 3) 150 years later; Galvani, as they showed, was right, Volta was wrong, but the story was not over. By means of the newly developed voltage clamp technique, which allowed them to control the voltage difference across the nerve membrane and measure the corresponding flow of ion current (Hodgkin \& Huxley, 1952a; Hodgkin \& Huxley, 1952b), the two English physiologists could tell the exact plot of the story (of excitability) without knowing the protagonists. These, the ion channel molecules, were indeed discovered only about 20 years later, and the molecular confirmation of the Hodgkin and Huxley $(\mathrm{HH})$ theory has been a further coup de théatre in the history of animal electricity. I will briefly summarize $\mathrm{HH}$ theory as it is known now, assuming therefore the knowledge of ion channels.

The cell membrane is electrically polarized: a difference of electrical potential $\left(\mathrm{V}_{\mathrm{m}}\right)$ exists between its intra- and extracellular face (of around $-80 \mathrm{mV}$ for example in neurons), being negatively charged inside and positively outside. Responsible for such polarization are ion channels, proteins embedded into the membrane lipid bi-layer and endowed with a pore, selectively permeable to ions, which are differently concentrated into and out of the cell. When, for example, a potassium ion tends to flow out through a channel along its concentration gradient (around $150 \mathrm{mM}$ inside and $4 \mathrm{mM}$ outside the cell), it leaves a negatively charged protein residue inside, which can-
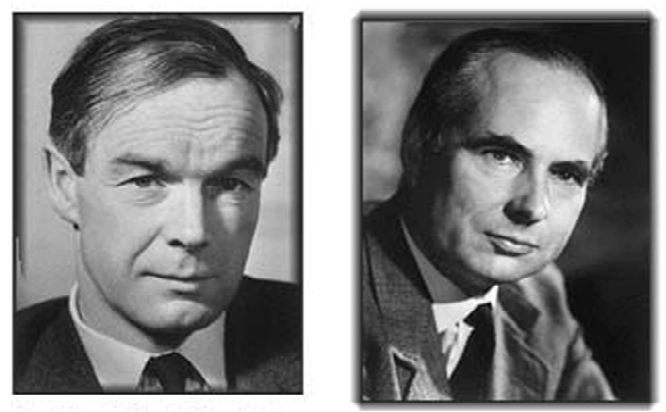

Figure 3.

Alan L. Hodgkin (1914-1998) and Andrew F. Huxley (1917-2012). They developed a mathematical model for the membrane excitability from their electrophysiological studies on nerve membranes. 
not permeate the channel's pore and which, in turn, operates an attractive electrical force on it. The condition when the work generated by the concentration gradient balances that of the electrical gradient is called electrochemical equilibrium of a given ion, and is described by the Nernst equation:

$$
E_{S}=\frac{R T}{z_{S} F} \ln \frac{[S]_{o}}{[S]_{i}}
$$

The pre-logarithmic factor (including the gas constant $R$, the temperature $T$ in Kelvin, the Faraday constant $F$, and the ion charge $z$ ) is constant at a given temperature: thus, if a membrane (biological or not) is selectively permeable to an ion $\mathrm{S}$ which is differently concentrated (i.e. the argument of logarithm is different from 1) across it, $V_{m}$ tends to assume an electrical polarity $\left(E_{S}\right)$, in general different from zero. Trans-membrane ion flow leads to a current that can be expressed, from Ohm's law, as:

$$
I_{S}=G_{S}\left(V_{m}-E_{S}\right)
$$

If channels are open (conductance $\left.G_{S} \neq 0\right)$, ion will flow $\left(I_{S} \neq\right.$ $0)$ until their Nernst equilibrium is reached $\left(V_{m}=E_{S}\right)$. Molecular pumps, driven by metabolic energy, maintain several ion gradients across the membrane and several families of ion channels permeable to them are present as well. $V_{m}$ is therefore determined by the combination of all the electrochemical gradients, each weighted by the corresponding permeability of the membrane to it. The total ion current can thus be written as:

$$
I_{\text {ion }}=I_{N a}+I_{K}+\cdots=G_{N a}\left(V_{m}-E_{N a}\right)+G_{K}\left(V_{m}-E_{K}\right)+\cdots
$$

Let's call $V_{r}$ the resting value of $V_{m}$. When $V_{m}$ value decreases, membrane is said to depolarize. When membrane restores $V_{r}$ value after depolarization, it is said to repolarize. When $V_{m}$ exceeds $V_{r}$, hyperpolarization is said to occur.

Hodking and Huxley knew that, when the nerve membrane was stimulated by injecting a brief (few ms) current pulse, if the amplitude of the pulse was large enough, $V_{m}$ rapidly depolarized, inverted its polarity of about $20 \mathrm{mV}$, and more slowly recovered $V_{r}$ value: this transient $V_{m}$ displacement, the action potential (AP), was first described by the German physiologist du Bois-Reymond in 1848. They also knew that depolarization was accompanied by sodium entry into and potassium exit from the cell. They found that the membrane permeability to ions (and therefore $\mathrm{G}_{\mathrm{S}}$ values) is voltage dependent, i.e. it changes when $V_{m}$ changes, and, what is more important, this voltage dependency is, in general, not instantaneous, but takes time to develop. Now we know that time dependency is due to the opening and closing properties of molecular gates that regulates ion trafficking through membrane channel pores (Figure 4).

Also, being made by a thin (few $\mathrm{nm}$ thick) insulating lipid bi-layer separating electrically charged compartments, the cell membrane per se can be represented as an electric capacitor of capacitance $\mathrm{C}$ (the parallelism with Leyden jars is striking). The current flowing through a capacitor is given by:

$$
I_{C}=C \frac{\mathrm{d} V_{m}}{\mathrm{~d} t}
$$

Thus, ion channels are embedded into the capacitive membrane, their conductance $G$ is voltage and time dependent and, when they are open, ion current flows according to Equation (4). All this can be incorporated into the equivalent electrical circuit reported in Figure 5. E terms represent the Nernst batteries for the different ion species (Equation (1)), and the G terms the corresponding electrical conductances (the arrows means "vol-

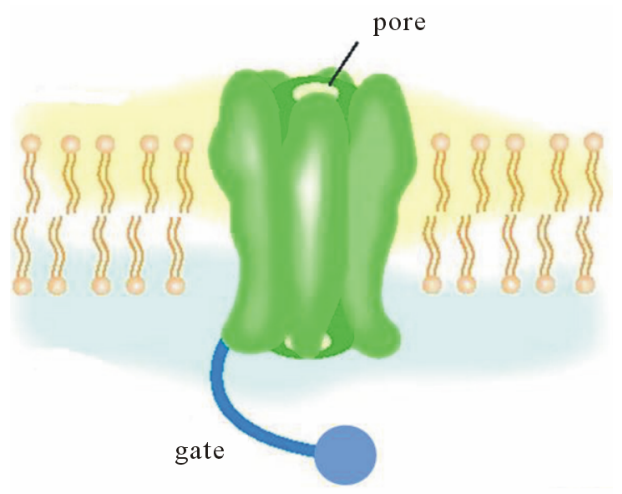

Figure 4.

Voltage dependent ion channel. An ion channel is a protein made by several sub-units that span the cell membrane and surround a central pore, which is selectively permeable to a given ion species. In the case of voltagedependent channels, the opening of the pore is regulated by $V_{m}$. Charged residues on the inner or outer side open or close following $V_{m}$ changes and making the voltagedependency time-dependent as well.

tage and time-dependent"). The total current flowing into the circuit (across the membrane) is therefore:

$$
I_{\text {tot }}=I_{C}+I_{\text {ion }}
$$

For a single cell, it is found that $I_{\text {tot }}$ is always zero, i.e. $I_{i o n}$ deposits all its ions on the membrane surface, causing $V_{m}$ to change and generate, according to Equations (5) and (6) an $\mathrm{I}_{\mathrm{C}}$ equal and opposite to $I_{i o n}$. This can be rewritten as:

$$
\frac{\mathrm{d} V m}{\mathrm{~d} t}=-\frac{I_{i o n}+I_{s t}}{C}
$$

where the constant term $I_{s t}$ can represent the external current injected by the electro-physiologist in order to study membrane properties, or it can simulate what happens in nature when neurons or muscular cells are stimulated by different biological mechanisms leading to current flow.

The HH theory explains that the voltage dependency of ion conductances $\left(G_{N a}, G_{K}\right.$, etc.) develops in a time dependent manner, due to the kinetics of the open probability $p$ of channels gates. When membrane depolarizes, some gates open (activation gates), other close (inactivation gates). An ion channel can be endowed with none, one, or $n$ activation and/or inactivation gates, and the corresponding open probability will be $p^{0}$, $p^{1}, p^{n}$ respectively, where:

$$
\frac{\mathrm{d} p}{\mathrm{~d} t}=\alpha_{p}\left(V_{m}\right)(1-p)-\beta_{p}\left(V_{m}\right) p
$$

Hodgkin and Huxley happen to work on a particularly simple biological membrane, that of the squid giant axon (Figure 6), where only three types of ion channels where presents: 1) Sodium channels with 3 activation gates (their open probability is classically called $\mathrm{m}$ ) and 1 inactivation gate (open probability is called h); 2) Potassium channels with 4 activation gates (n) and none inactivation gates; 3 ) Leakage channels, no activation nor inactivation gates, and with a constant (ohmic) conductance. They derived the constant terms $\mathrm{G}$ and the exponential functions $\alpha_{p}\left(V_{m}\right)$ and $\beta_{p}\left(V_{m}\right)$ for $\mathrm{Na}$ and $\mathrm{K}$ conductances with a series of elegant voltage clamp experiments (Hodgkin \& Huxley, 1952a; Hodgkin \& Huxley, 1952b), and summarized the membrane dynamics into the following equation system: 


$$
\left\{\begin{array}{l}
C \frac{\mathrm{d} V_{m}}{\mathrm{~d} t}=I_{s t}-G_{N a} m^{3} h\left(V_{m}-E_{N a}\right)-G_{K} n^{4}\left(V_{m}-E_{K}\right)-G_{L}\left(V_{m}-E_{L}\right) \\
\frac{\mathrm{d} m}{\mathrm{~d} t}=\alpha_{m}\left(V_{m}\right)(1-m)-\beta_{m}\left(V_{m}\right) m \\
\frac{\mathrm{d} h}{\mathrm{~d} t}=\alpha_{h}\left(V_{m}\right)(1-h)-\beta_{h}\left(V_{m}\right) h \\
\frac{\mathrm{d} n}{\mathrm{~d} t}=\alpha_{n}\left(V_{m}\right)(1-n)-\beta_{n}\left(V_{m}\right) n \\
\alpha_{m}\left(V_{m}\right)=f_{\alpha m}\left(V_{m}\right) \\
\beta_{m}\left(V_{m}\right)=f_{\beta m}\left(V_{m}\right) \\
\alpha_{h}\left(V_{m}\right)=f_{\alpha h}\left(V_{m}\right) \\
\beta_{h}\left(V_{m}\right)=f_{\beta h}\left(V_{m}\right) \\
\alpha_{n}\left(V_{m}\right)=f_{\alpha n}\left(V_{m}\right) \\
\beta_{n}\left(V_{m}\right)=f_{\beta n}\left(V_{m}\right)
\end{array}\right.
$$

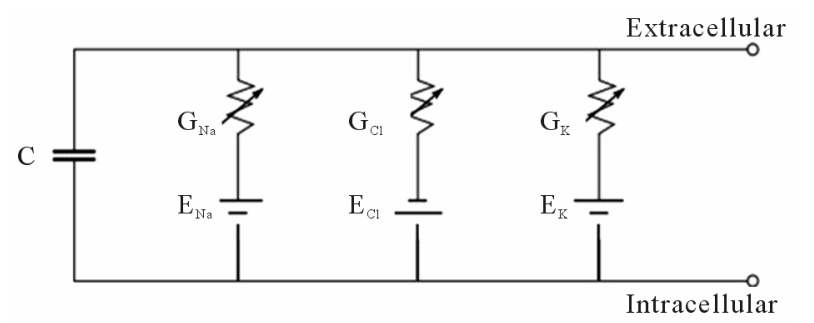

Figure 5.

Electrical equivalent circuit for an excitable membrane. Cell membrane can be considered as a parallel combination of a capacitor $\mathrm{C}$, representing dielectric properties of the lipid bi-layer, and several ion conductances $\mathrm{G}$, in series with the corresponding Nernst batteries.

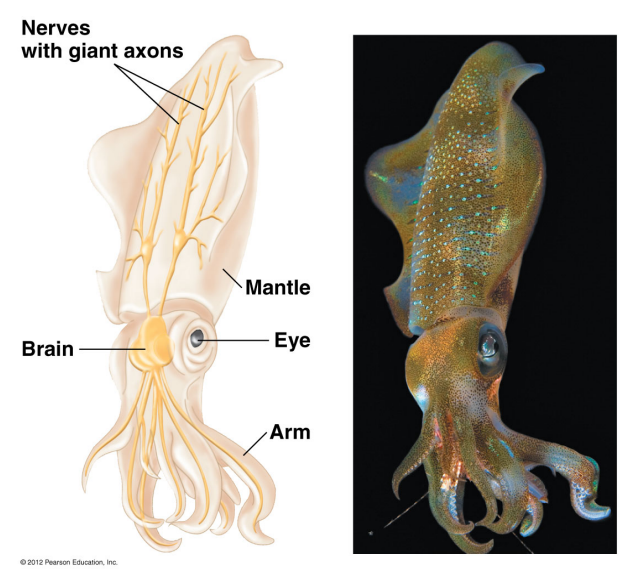

Figure 6.

The squid giant axon. The giant axon is a very large (up to $1 \mathrm{~mm}$ in diameter) and long (several $\mathrm{cm}$ ) projection of a single neuron that controls water propulsion in this cephalopods. (image taken from Memorial University at http://www.mun.ca/biology/).

This is an ordinary differential equation (ODE) system. Whereas the solution of an algebraic equation system is a given set of numbers, the solution of an ODE system is a set of functions of the differentiated variables. When the embedded constants are accurately derived from in vivo experiments and the system "stimulated" with appropriate $I_{s t}$ values, its solution provides a set of functions, including the AP and the time course of the corresponding ion conductances (Figure 7). The system reproduces the dynamics of the excitable nerve and allow to dissect the underlying mechanisms. When $V_{t h}$ is reached, $I_{N a}$ dynamics becomes auto-regenerative: sodium channels (their activation gates) start to open abruptly and let sodium ions enter the cell (following Equation (2)). Sodium entry depolarizes the membrane (towards $E_{\mathrm{Na}}$ ) and depolarization, in turn, open more $\mathrm{m}$ gates and lets more ions in. All this happens in less than $1 \mathrm{~ms}$, while AP reaches its peak. Eventually h gates, also triggered by depolarization, close sodium channels. At the same time depolarization also open $n$ gates of potassium channels. Potassium ions rapidly exit the cell following their electrochemical gradient and repolarise the membrane (towards $\mathrm{E}_{\mathrm{K}}$ ). The entire AP waveform lasts (in the case of the squid giant axon) about 2 - $3 \mathrm{~ms}$ (Figure 7). Action potentials underlie nervous activity, and trigger cardiac and skeletal muscular contraction in higher organism; they are also present in prokaryotes, protists, green algae, and practically in any form of life, when the cell generates an all-or-none response to any sort of stimuli coming from its inside or outside environment. For more information on HH theory see (Hille, 2001; Aidley, 1998).

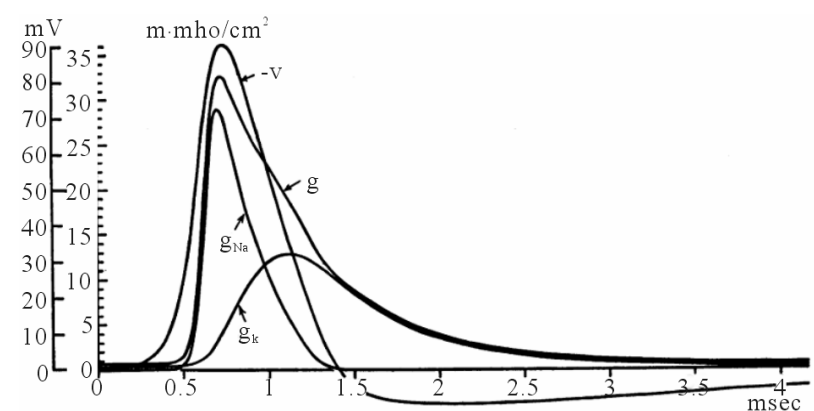

Figure 7.

$\mathrm{HH}$ action potential model. Numerical solution of $\mathrm{HH}$ model showing sodium and potassium components of membrane conductance $(\mathrm{g})$ during action potential $(-\mathrm{V})$. Note that $V_{m}$ scale was shifted vertically in order to make $V_{r}=0$. (From HH original paper: Hodgkin \& Huxley, 1952a). 


\section{Computational Solutions of HH Systems}

Since the time of its formulation, the $\mathrm{HH}$ equation system has been a powerful tool to reproduce and study membrane excitability. Unfortunately, the solutions of the system, functions of $V_{m}$ and time (later came also space, i.e. spatial conduction of excitability), cannot be written analytically. No discrete combinations of $\sin \left(V_{m}, t\right), \exp \left(V_{m}, t\right)$, nor any analytical function can, in general, describe the time course of the AP, nor that of the underlying ion currents. Numerical computation, based essentially on Euler's method (Borse, 1997), allow to do the job; such methods have preceded the computer technology but greatly advanced with it. The principles of Euler's integration of ODE, like those of $\mathrm{HH}$, can easily be thought in high schools; I will not discuss them here but note that their knowledge is not irrelevant for "computationally thinking" many physiological problems. The computing steps needed to solve ODE systems are nowadays incorporated into easy-to-handle routines that can be run from many mathematical computing environment. One of the most popular for both teaching and research purposes is definitely Matlab (MathWorks, Natick, Massachusetts, USA), where commands to solve the $\mathrm{HH}$ system can be written into straightforward scripts, and solutions obtained by means of built-in ODE solvers. Equations system (9) can be re-written into the following Matlab script:

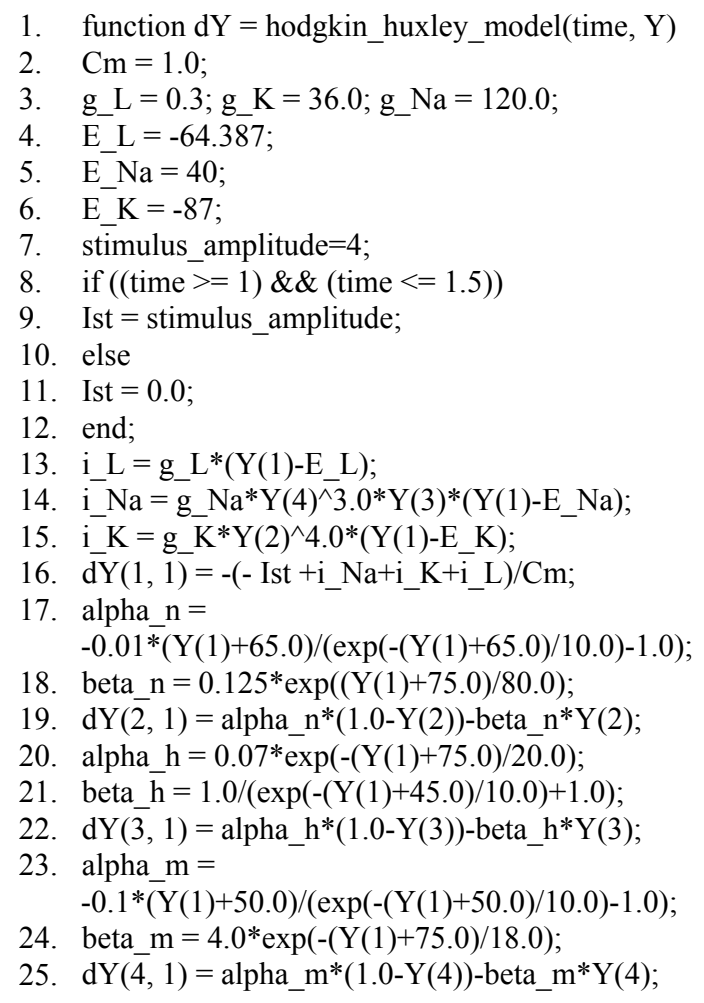

Line 1 defines the name of this script ("hodgkin_huxley_ model") which, in Matlab language, is considered as a "function". Lines 2 - 12 define membrane capacitance (I will not discuss here scaling and dimensional details), ion channels maximum conductance values, amplitude and timing of the current stimulus $I_{s t}$ (amplitude $=4$, starts at time $1 \mathrm{~ms}$ and stops at $1.5 \mathrm{~ms}$, lasting therefore $0.5 \mathrm{~ms}$ ). Remaining lines report explicitly the exponential functions for $\alpha$ and $\beta$ described above for each gate and their dynamics (Equation (8)). Line 13 - 16 contain Equation (7). The function "hodgkin huxley model" will be called from a second script. I name it "main_HH"and report it here:

1. $\mathrm{tMax}=10$;

2. $\mathrm{Y}=[-76.0,0.325,0.6,0.05]$;

3. options $=$ odeset('reltol',1e-8,'abstol', $\left.\left[1^{-8}, 1^{-8}, 1^{-8}, 1^{-8}\right]\right)$;

4. [tData, YData]=ode15s(@hodgkin_huxley_model, [0 tMax], Y, options);

Line 1 sets duration of simulation in $\mathrm{ms}$, line 2 initial conditions, lines 3 and 4 call the Matlab ODE solver "ode15s" and make it to solve the equations listed in the script "hodgkin_huxley_model". When, from the Matlab prompt, we simply type "main_HH", the system will be solved numerically (note that simulating $10 \mathrm{~ms}$ of membrane dynamics was taking about a week for the 1950's analog computers, it takes much less than $1 \mathrm{~s}$ now on my laptop). The ODE solver generates in this case a vector tData which is the time of the simulation, and a 4 columns matrix called YData, where the time courses of $V_{m}, m$, $h$, and $n$ are reported in columns 1 to 4 respectively. These 5 vectors contain all we need to know about AP dynamics. We can try simulating the application of a sodium channel blocker known (from experiments) to reduce sodium conductance by $50 \%$, simply multiplying g_Na by 0.5 , or see what happens when some genetic disease leads to a $30 \%$ increase in potassium channel conductance (simply multiply g_K by 1.3 ). We have just to type: "plot(tData, YData(:,1))" and Matlab will produce a graphic representation of the AP. We can type: "plot(tData, YData(:,2))" to see the time course of m; or, again, we can type:

\section{i_Na=g_Na*YData(:,4).^3.0.*YData(:,3).*(YData(:,1)-E_Na); plot(tData,i_Na);}

to see the time course of Sodium current (note that when simple algebraic operation are applied to vectors, a dot should be placed between the vector and the operator). Same for potassium or leakage current. Ready to use scripts containing HHtype equations systems like that discussed here can be found on different web sources, like http://cor.physiol.ox.ac.uk., or directly from the MathWorks website at

http://www.mathworks. com/matlabcentral/fileexchange.

\section{Back to the Controversy}

"In order to save something of the Galvani's hypothesis on animal electricity, which I consider to be wrong through a number of experimental evidences, and which I have replaced with the principle of a purely artificial electricity where the cause is all extrinsic to the animal, it would be necessary that the followers of Galvani showed me contractions in frogs elicited by arcs made by metals of the same species; they will never be able to do so". These are Volta's words from a letter to a colleague in 1795 (Piccolino \& Bresadola, 2003: p. 391). Too bad at his time he could not experiment on the electrical activity of cardiac pacemaker cells, nor on the rhythmic firing of pacemaker neurons, where the electrical excitability develops without any external stimulus. In the HH model that I reported above, the external stimulus is indicated by $I_{s t}$. In the Galvani-Volta bi-metallic arc experiment, it was the "battery-like" 
nature of the arc that made $I_{s t}$ flow across the membrane. $I_{s t}$ elicited in turn an AP on the crural nerves, and the AP was then conducted to the muscle where it triggered contraction. We can play around with $I_{s t}$ in the model, and see where the core of the controversy is hidden. If we go to the script "hodgkin huxley_model" in Matlab, we set stimulus_amplitude $=4$, and we run main_HH, we obtain the sub-threshold voltage trace reported in Figure 8. Vm slightly depolarizes (about $2 \mathrm{mV}$ ) during the current injection, and slowly recovers its $V_{r}$ value after injection stops. We can try different amplitudes $(-8,-4,+4,+8$, +12 ) and obtain $V_{m}$ deflections reaching each time a value (at time $1.5 \mathrm{~ms}$, when the pulse ends) proportional to the injected current (Figure 9): membrane is, in this $V_{m}$ range, ohmic, i.e. it behaves like a passive RC circuit. Now, if we give $I_{s t}$ a value of 16 and we run main_HH, $V_{m}$ reaches its threshold value $V_{t h}$ (arrow in figure) and an AP is elicited (Figure 10). The intrinsic nature of AP is all-or-none, i.e. it does not depend on the stimulus, as we can easily verify by playing around with higher $I_{s t}$ values. During the AP the membrane dissipate energy asso-

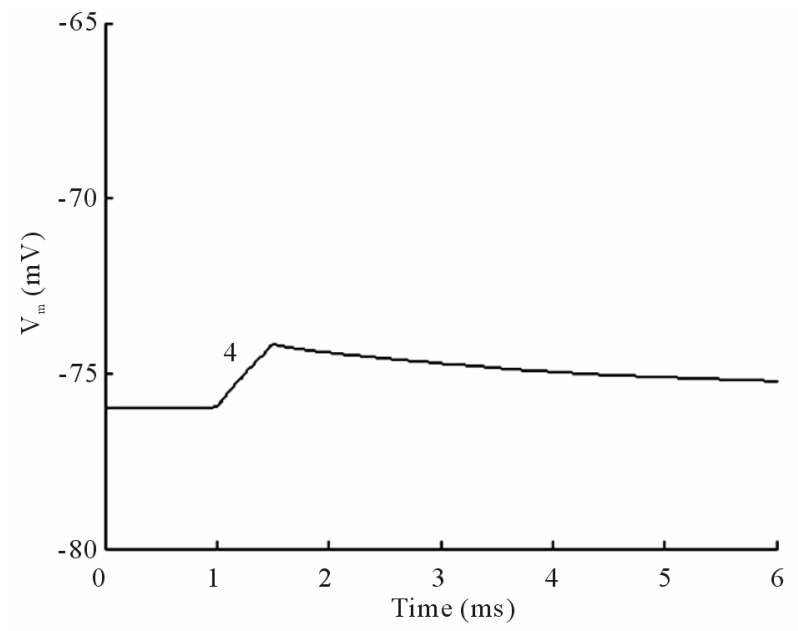

Figure 8.

Sub-threshold voltage response. The trace is obtained by setting stimulus_amplitude $=4$ in the Matlab script.

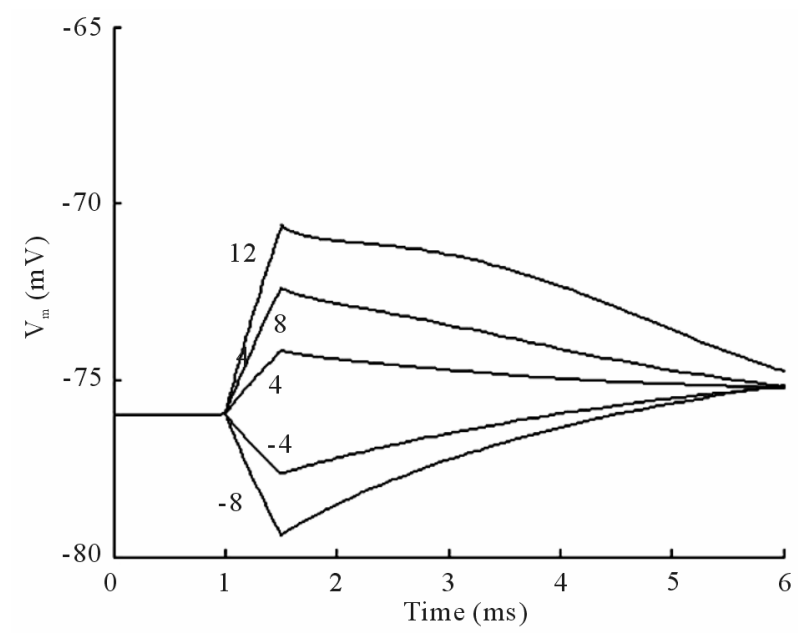

Figure 9.

More sub-threshold traces. Stimulus_amplitude was set, in turn, to $-8,-4$, $+4,+8,+12$.
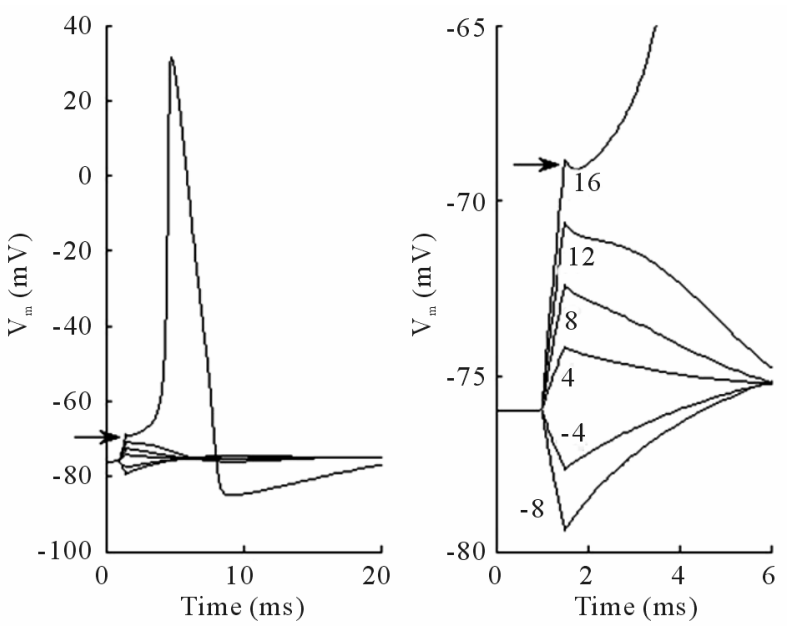

Figure 10.

Sub- and supra-threshold responses. When stimulus_amplitude is set to 16, $V_{t h}$ (arrow) is reached and an AP elicited. Full scale on the left panel, and expanded scale on the right.

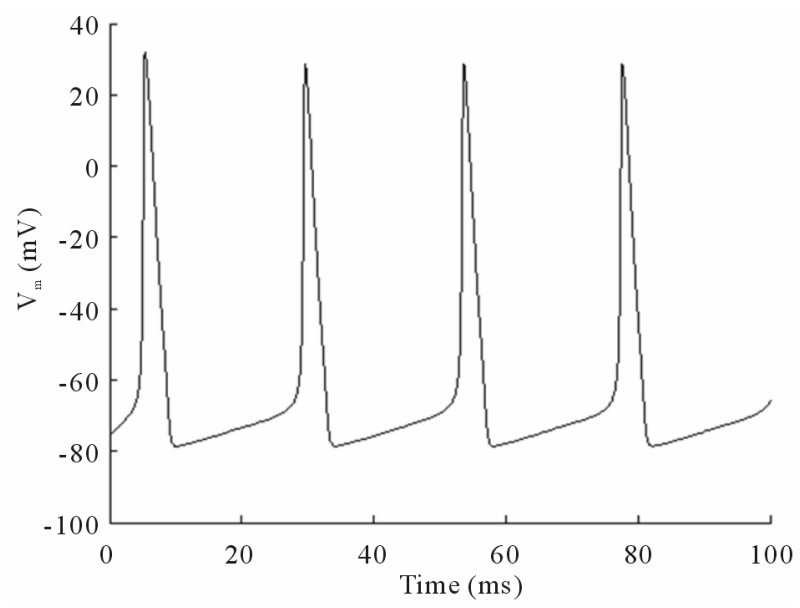

Figure 11.

Spontaneous activity. It is enough to slightly increase extracellular potassium (i.e. setting $\mathrm{EK}=-80 \mathrm{mV}$ ) for inducing spontaneous pacemaker activity even in the absence of any external stimulus (stimulus_amplitude = $0)$.

ciated to the electrochemical gradients that sustain its electrical polarity. This gradients are maintained by ion pumps and other molecular transport mechanisms. Gradients (and ion pumps as well) can also be incorporated into the Matlab script by assigning physiological values to $[\mathrm{Na}+] \mathrm{o},[\mathrm{Na}+] \mathrm{i},[\mathrm{K}+] \mathrm{o},[\mathrm{K}+] \mathrm{i}$, and explicitly adding equation (1) for each ion species. In our simplified script though, gradients and their required metabolic energy are all contained into lines $4-6$. Also, we have focused here only on nerve excitability. The membrane of the muscle cell is excitable as well and transduces AP into mechanical activity through a mechanism known as excitation-contraction coupling, which is also included with its dynamics into very recent and more complex mathematical models.

In conclusion, it is useful to look at the bi-metallic arc experiment and, through it, at the all controversy, from the Matlab script of the HH model. We can observe that the Haller's irritability lies into the potential energy associated with metaboli- 


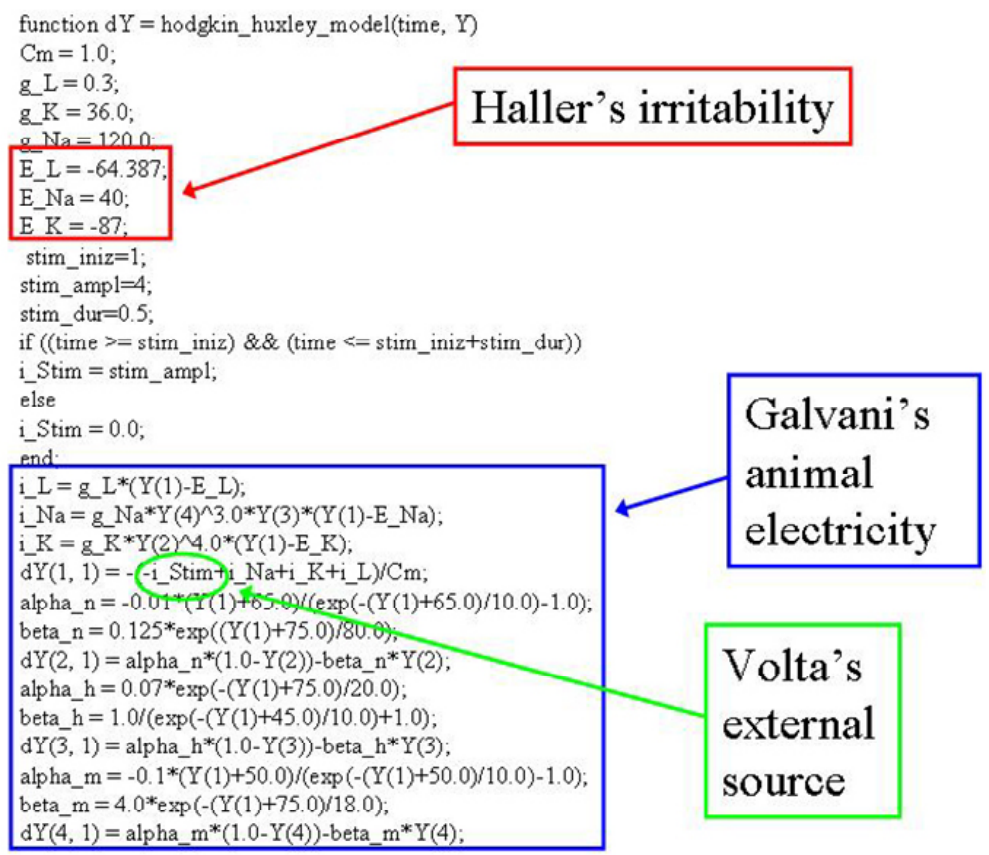

Figure 12.

The key points of the controversy can be discussed by playing around with the Matlab script of the HH model.

cally maintained electrochemical gradients (ENa, EK, etc.), the Galvani's excitability is indeed given by the flux of an "animal electricity" (Itot) through ion channels, as their non-ohmic dynamics (voltage and time dependency of $m, h$ and $n$ ) is triggered. The external intervention, the trigger, is present in the model through the $I_{s t}$ term, what Volta thought as the source of the all phenomenon and which, in the bi-metallic arc experiment, was indeed due to the current flow driven by the different electro-negativity of metals. As mentioned above, pacemaker activity of some excitable membranes is the clearest example of how Volta's thinking was wrong. We can make $I_{s t}$ equals zero, i.e. we can completely turn off the stimulus (Volta was suggesting so in the words that opens this paragraph) and simply simulate a slight change in potassium gradient across the membrane (equation 1) resulting in a $7 \mathrm{mV}$ depolarization of EK (hyperkalemic conditions are not uncommon in physiology and pathology). We do so by assigning a value of -80 instead of -87 to EK into the script "hodgkin_huxley_model". We obtain that the simulated membrane generates APs spontaneously and repetitively (Figure 11). Again, we can play around with EK value and see how pacemaker activity changes accordingly. Automatic firing is achieved in nature thanks to the presence of particular ion channels expressed in pacemaker cells (they generate the spontaneous heart beat and a number of fundamental neurological functions), which are not included in the original $\mathrm{HH}$ model, but this is not the point here. The point is that excitability is indeed a form of "animal electricity"; the cell membrane contains the fuel necessary to the AP (ion gradients) and the engine to generate it (ion channels); there are conditions in which an external current $\left(I_{s t}\right)$ is required for the process to take place, but this is not necessarily required (see scheme in Figure 12).

Computational techniques are increasingly gaining importance into modern biological (Fall, Marland, Wagner, \& Tyson,
2010) and physiological (Keener \& Sneyd, 2008; Zaniboni, 2012) research and, according to my experience, they can be successfully approached also in high schools and in undergraduate courses. What a mathematical model does is to translate our logical knowledge of a phenomenon into a synthetic series of commands; in doing so, the $\mathrm{HH}$ model of the AP is shown here to be a useful tool for removing ambiguities that still concern one of the greatest controversies in the history of science. The study of this controversy can also be a great opportunity to combine history, physiology and technology into an interdisciplinary dialog fruitful for both teachers and students

\section{REFERENCES}

Aidley, D. J. (1998). The physiology of excitable cells (4th ed.). Cambridge: Cambridge University Press. doi:10.1017/CBO9781139171182

Bernardi, W. (2001). The controversy over animal electricity in 18thcentury Italy: Galvani, Volta, and others. Revue d'Histoire des Sciences, 54, 53-70.

Borse, G. J. (1997). Numerical methods with Matlab. Boston, MA: PWS Publishing Company.

Bresadola, M. (2008). Animal electricity at the end of the eighteenth century: The many facets of a great scientific controversy. Journal of the History of the Neurosciences: Basic and Clinical Perspectives, 17, 8-32. doi:10.1080/09647040600764787

Fall, C. P., Marland, E. S., Wagner, J. M., \& Tyson, J. J. (2010). Computational cell biology. New York: Springer-Verlag, Inc.

Hille, B. (2001). Ionic channels of excitable membranes (3rd ed.). Sunderland, MA: Sinauer Associates Inc.

Hodgkin, A. L., \& Huxley, A. F. (1952a). The dual effect of membrane potential on sodium conductance in the giant axon of Loligo. The Journal of Physiology, 116, 497-506.

Hodgkin, A. L., \& Huxley, A. F. (1952b). A quantitative description of membrane current and its application to conduction and excitation in nerve. The Journal of Physiology, 117, 500-544. 


\section{ZANIBONI}

Keener, J., \& Sneyd, J. (2008). Mathematical physiology. New York: Springer-Verlag, Inc.

Piccolino, M. (1997). Luigi Galvani and animal electricity: Two centuries after the foundation of electrophysiology. Trends in Neurosciences, 20, 443-448. doi:10.1016/S0166-2236(97)01101-6

Piccolino, M. (1998). Animal electricity and the birth of electrophysiology: The legacy of Luigi Galvani. Brain Research Bulletin, 46,
381-407.

Piccolino, M., \& Bresaola, M. (2003). Frogs, electric rays, and sparks. Galvani, Volta, and the animal electricity. Turin: Bollati Boringhieri. Zaniboni M. (2012) Late phase of repolarization is autoregenerative and scales linearly with action potential duration in mammals ventricular myocytes: A model study. IEEE Transactions on Biomedical Engineering, 59, 226-233. doi:10.1109/TBME.2011.2170987 